\title{
Prognostic and clinicopathological significance of FGFR1 gene amplification in resected esophageal squamous cell carcinoma: a meta-analysis
}

\author{
Yan Wang", Yanming Wu", Jialong Li", Yutian Lai, Kun Zhou, Guowei Che \\ Department of Thoracic Surgery, West China Hospital, Sichuan University, Chengdu, 610041, China \\ Contributions: (I) Conception and design: G Che; (II) Administrative support: None; (III) Provision of study materials or patients: None; (IV) \\ Collection and assembly of data: Y Wang, Y Wu, J Li; (V) Data analysis and interpretation: Y Lai, K Zhou; (VI) Manuscript writing: All authors; (VII) \\ Final approval of manuscript: All authors. \\ "These authors contributed equally to this work. \\ Correspondence to: Guowei Che. Department of Thoracic Surgery, West China Hospital, Sichuan University, Guoxuexiang No. 37, Chengdu 610041, \\ China. Email: cheguowei_hx@aliyun.com.
}

Background: Previous studies about the prognostic and clinicopathological significance of fibroblast
growth factor receptor 1 (FGFR1) amplification in resected esophageal squamous cell carcinoma (ESCC) are
controversial. Therefore, the aim of the current meta-analysis was to determine the association of FGFR1
amplification with prognosis and clinicopathological characteristics of resected ESCC patients.
Methods: The PubMed, EMBASE, Web of Science, The Cochrane Library, CNKI, Wanfang, VIP and
SinoMed databases were searched systematically from the establishment date of databases to April 1, 2019
to identify related studies. The correlations of FGFR1 amplification of prognosis and clinicopathological
characteristics in ESCC were assessed by the combined hazard ratio (HR) with 95\% confidence interval (CI)
and combined odds ratio (OR) with $95 \%$ CI, respectively. All statistical analyses were performed by the Stata
12.0 software.

Results: A total of nine retrospective studies involving 2,326 patients who received the surgery were included into the current meta-analysis. The results indicated that FGFR1 amplification was significantly correlated with worse overall survival (OS) ( $\mathrm{HR}=1.50$, 95\% CI: 1.25-1.81, $\mathrm{P}<0.001$ ), disease-free survival (DFS) (HR =1.58, 95\% CI: 1.27-1.96, $\mathrm{P}<0.001)$, lymph node metastasis (OR =1.45, 95\% CI: 1.13-1.86, $\mathrm{P}=0.004)$, higher $\mathrm{TNM}$ stage $(\mathrm{OR}=1.33,95 \% \mathrm{CI}: 1.03-1.72, \mathrm{P}=0.027)$ and poorer differentiation $(\mathrm{OR}=1.10$, 95\% CI: $1.07-1.13, \mathrm{P}<0.001)$.

Conclusions: The current meta-analysis strongly demonstrates that FGFR1 amplification is an independent prognostic risk factor for resected ESCC patients and more prevalent among patients with advanced tumor stage and poorer differentiation.

Keywords: Fibroblast growth factor receptor 1 (FGFR1); esophageal squamous cell carcinoma (ESCC); surgery; prognosis; clinicopathological characteristics; meta-analysis

Submitted Jul 18, 2019. Accepted for publication Sep 24, 2019.

doi: $10.21037 / \mathrm{atm} .2019 .10 .69$

View this article at: http://dx.doi.org/10.21037/atm.2019.10.69

\section{Introduction}

In esophageal squamous cell carcinoma (ESCC), tyrosine kinase inhibitors (TKIs) mainly target the vascular endothelial growth factor receptor (VEGFR) and epidermal growth factor receptor (EGFR), which displays important significance in clinical practice (1). The FGFR1 is one member of the FGFR family which consists of four tyrosine kinase receptors: FGFR1, FGFR2, FGFR3 and 
FGFR4 and they have been found to be another potential target of TKIs. Actually, their inner structures are similar to those of some pharmacologic therapeutic targets such as VEGFRs, EGFRs and platelet-derived growth factor receptors (PDGFRs) (2); which may indicate their great clinical value.

According to previous reports, FGFRs could initiates many intracellular events to activate major proliferative and survival signal pathways; furthermore, they could affect many biological processes such as the neovascularization, wound repair and embryonic development (3). In recent years, increasing reports manifested that FGFRs play a key role in the tumorigenesis and development of several cancers (4-6). Among three main deregulation form, including the point mutation, translocation and amplification, the amplification is the most common one.

FGFR1 gene amplification has been demonstrated to show high prognostic value in several kinds of cancers such as squamous cell lung cancer (7), head and neck squamous cell carcinoma (8) and breast cancer (9). Besides, Xie et al. (10) proved that FGFR1 amplification was significantly correlated with some clinicopathological characteristics like the smoking, sex and histology in non-small cell lung cancer (NSCLC), especially in squamous cell cancer (SCC). However, its association with prognosis and clinical pathological parameters of ESCC patients remains unclear now. Although there are already several studies which explored clinical significance of FGFR1 amplification in resected ESCC, their results are different among each other (11-19).

Therefore, we conducted this meta-analysis to further determine the correlation of FGFR1 amplification with survival and clinicopathological characteristics of ESCC patients who underwent the operation and contribute to clinical application of FGFR1.

\section{Methods}

\section{Literature search}

We searched the PubMed, EMBASE, Web of Science, Cochrane Library, CNKI, VIP, Wanfang and SinoMed databases for related articles published from the establishment date of databases to April 1, 2019 with the following terms "FGFR1", "fibroblast growth factor receptor 1", "esophageal", "esophagus", "tumor", "cancer", "carcinoma" and "neoplasm". Besides, the references cited in the included studies were also identified for eligibility.

\section{Inclusion criteria and exclusion criteria}

Inclusion criteria were: (I) patients were diagnosed as ESCC pathologically and the amplification of FGFR1 was detected by the monoclonal antibody (MA), fluorescent in situ hybridization (FISH) or quantitative reverse transcription polymerase chain reaction (QRT-PCR); (II) the studies described the association of FGFR1 amplification with ESCC patient prognosis [overall survival (OS) or diseasefree survival (DFS)] using the hazard ratio (HR) with $95 \%$ confidence interval (CI) or by Kaplan-Meier curves and clinicopathological characteristics of patients; (III) all patients receive the surgical therapy; (IV) the articles were published with full-texts; (V) if the data were duplicated or overlapped, only the most recent publication was included; (VI) articles were written in English or Chinese.

Exclusion criteria were as following: (I) letters, reviews, animal trials, meeting abstracts and case reports; (II) articles did not provide enough information to calculate the HR with $95 \%$ CI when HRs with $95 \%$ CIs for survival and Kaplan-Meier curves were not reported.

The literature selection was performed by two independent authors (Y Wang and $\mathrm{Y}$ Wu) and disagreements were resolved by discussion.

\section{Data extraction and quality assessment}

The data were extracted using an excel sheet (Microsoft Corporation) and the following information were collected: name of the first author, publication year. Country, number of patients with FGFR1 amplification, gender, age, tumor depth, lymph node metastasis, TNM stage, differentiation status, drinking history, smoking history, treatment method, detection method, definition of FGFR1 amplification, clinical outcomes, source of HR and HR with $95 \%$ CI.

In our study, the Newcastle-Ottawa quality assessment scale (NOS) was applied for the quality evaluation of included publications (20). Studies earning a score of 6 or higher were regarded as high-quality studies.

The process of data extraction and quality evaluation was also performed by two researchers ( $\mathrm{Y}$ Wang and $\mathrm{Y} \mathrm{Wu}$ ) independently.

\section{Statistical analysis}

Correlations of FGFR 1 gene amplification with clinicopathological parameters of resected ESCC patients were estimated by the pooled odds ratios (ORs) with $95 \%$ 


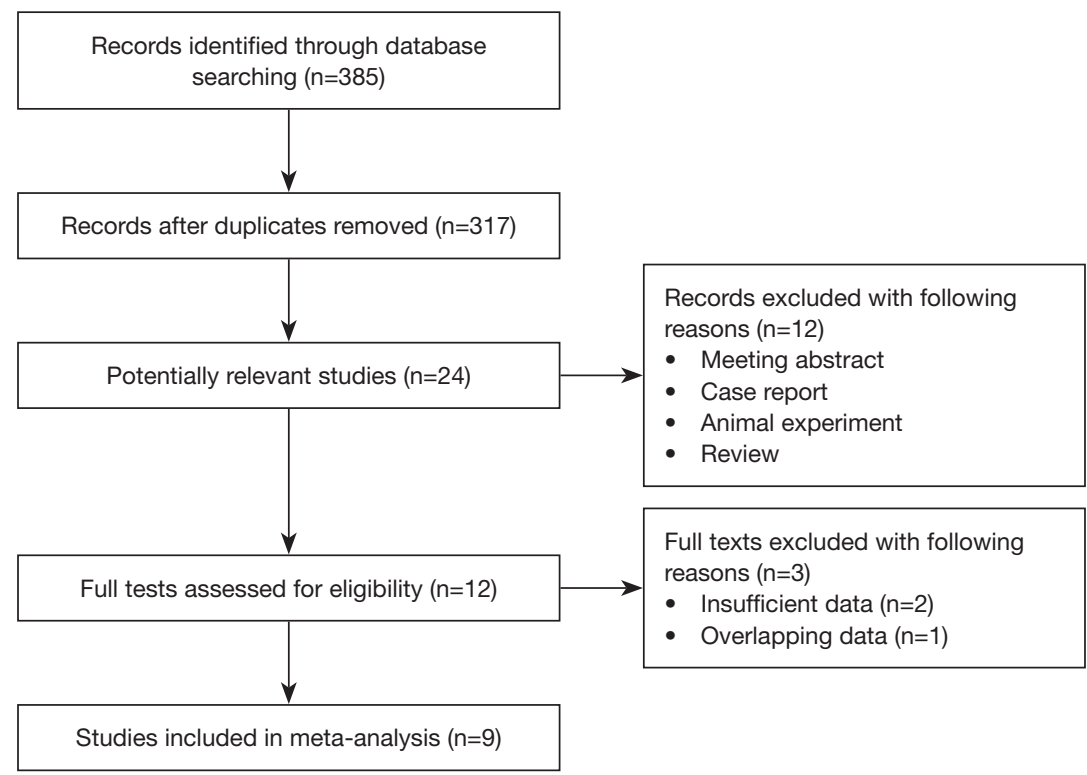

Figure 1 Flow diagram of the literature review.

CIs and correlations between FGFR1 amplification and prognosis were assessed by the pooled HRs with $95 \%$ CIs. HRs from multivariate models were applied whenever available; if they were not reported directly, then they would be calculated from Kaplan-Meier curves with the method described by Tierney et al. (21). The heterogeneity among included studies was calculated using the Chi-square based Q-test and $\mathrm{I}^{2}$ statistic (22). When significant heterogeneity was observed representing as $\mathrm{P}<0.10$ or $/$ and $\mathrm{I}^{2}>50 \%$, the random effect model was applied to calculate the ORs and HRs with corresponding 95\% CIs; otherwise the fixed effect model was adopted (23). Subgroup analyses based on the country and detection method were performed to explore the influence of these two factors on the prognostic value of FGFR1 gene amplification in ESCC or potential causes of heterogeneity; and the sensitivity analysis was conducted to assess the stability of the pooled results. Begg's funnel plot and Egger's test were used to assess potential publication bias (24). $\mathrm{P}$ values $<0.05$ were considered significant and all the statistical analyses were conducted using the Stata 12.0 software (Corporation, TX, USA).

\section{Results}

\section{Literature search process and basic characteristics of included studies}

Specific flow diagram of the current meta-analysis was shown in Figure 1. A total of 9 retrospective studies (11-19) involving 2,326 resected ESCC patients were enrolled eventually according to the criteria and exclusion criteria. Among included patients, 381 (16.4\%) patients were with FGFR1 gene amplification; and the ratios of positive FGFR1 amplification in included articles ranged from $8.6 \%$ to $64.9 \%$. All patients received the surgery and all included studies reported the relation of FGFR1 amplification with OS in ESCC. In most of the included studies (6/9), the FISH method was applied to measure the status of FGFR1 gene amplification; the MA and QRT-PCR methods were used in 2 and 1 studies, respectively. Other detailed information was presented in Table 1 .

\section{Correlations of FGFR1 amplification with prognosis in resected ESCC}

The results of meta-analyses for OS demonstrated that FGFR1 amplification was an independent risk factor for OS of ESCC patients (HR $=1.50,95 \%$ CI: $1.25-1.81$, $\mathrm{P}<0.001)$ with low heterogeneity $\left(\mathrm{I}^{2}=3.3 \%, \mathrm{P}=0.407\right)$ (Figure 2). Subgroup analyses for OS based on the country and detection method further verified above results with one exception (Table 2). There was no significant relation between FGFR1 amplification and poor OS in ESCC patients who were detected for FGFR1 amplification status with the MA method (HR $=0.64,95 \%$ CI: 0.34-1.22, 
Table 1 Basic characteristics of included studies

\begin{tabular}{|c|c|c|c|c|c|c|c|c|c|c|}
\hline Author & Year & Country & $\begin{array}{l}\text { Sample } \\
\text { size }\end{array}$ & $\begin{array}{l}\text { Positive, } \\
\text { n (\%) }\end{array}$ & $\begin{array}{l}\text { TNM } \\
\text { stage }\end{array}$ & $\begin{array}{l}\text { Detection } \\
\text { method }\end{array}$ & Definition of FGFR1 amplification & Outcome & $\begin{array}{l}\text { Source } \\
\text { of HR }\end{array}$ & $\begin{array}{l}\text { NOS } \\
\text { score }\end{array}$ \\
\hline $\begin{array}{l}\text { Sugiura } \\
\mathrm{K}(11)\end{array}$ & 2007 & Japan & 79 & $47(59.5)$ & I-IV & MA & $\begin{array}{l}\text { Stained cytoplasm of cancer cells } \\
>30 \%\end{array}$ & OS & $E$ & 7 \\
\hline $\begin{array}{l}\text { Wang } \\
\text { D (12) }\end{array}$ & 2014 & China & 82 & $13(15.9)$ & $|-|||$ & $\mathrm{FISH}$ & $\begin{array}{l}\text { FGFR } 1 \text { copy number } \geq 6 \text { or FGFR1/ } \\
\text { CEN8 ratio } \geq 2.0\end{array}$ & OS & $\mathrm{R}$ & 8 \\
\hline $\begin{array}{l}\text { Shimada } \\
\text { Y (14) }\end{array}$ & 2015 & Japan & 57 & $37(64.9)$ & I-IVa & MA & $\begin{array}{l}\text { Sores for intensity and distribution } \\
\text { of expression } \geq 4\end{array}$ & OS & $\mathrm{R}$ & 7 \\
\hline $\begin{array}{l}\text { Wang } \\
\text { D (17) }\end{array}$ & 2017 & China & 556 & $67(12.1)$ & $|-| I \mid$ & FISH & $\begin{array}{l}\text { FGFR1 copy number } \geq 6 \text { or FGFR1/ } \\
\text { CEN8 ratio } \geq 2.2\end{array}$ & OS/DFS & $\mathrm{R}$ & 7 \\
\hline $\begin{array}{l}\text { Song } \\
Q(18)\end{array}$ & 2017 & China & 506 & $44(8.7)$ & I-IV & FISH & $\begin{array}{l}\text { FGFR } 1 \text { copy number } \geq 6 \text { or FGFR1/ } \\
\text { CEN8 ratio } \geq 2.0 \text { or percentage of } \\
\text { tumor cells containing } \geq 15 \text { or large } \\
\text { cluster in } \geq 10 \% \text { cells }\end{array}$ & OS/DFS & $\mathrm{R}$ & 7 \\
\hline $\begin{array}{l}\text { Chen } \\
\text { B (19) }\end{array}$ & 2018 & China & 145 & $73(50.3)$ & $\|-I I I$ & QRT-PCR & Median mRNA expression & OS/DFS & $\mathrm{R}$ & 7 \\
\hline
\end{tabular}

TNM, tumor-node-metastasis; MA, monoclonal antibody; FISH, fluorescent in situ hybridization; QRT-PCR, quantitative reverse transcription polymerase chain reaction; FGFR1, fibroblast growth factor receptor 1; CEN, centromere; OS, overall survival; DFS, diseasefree survival; HR, hazard ratio; R, reported; E, estimated; NOS, Newcastle-Ottawa quality assessment scale.

$\mathrm{P}=0.175)$ without any heterogeneity $\left(\mathrm{I}^{2}=0.0 \%, \mathrm{P}=0.742\right)$.

Four articles involving 1,733 patients explored the predictive role of FGFR1 amplification on DFS in ESCC. The combined HR (95\% CI) was 1.58 (1.27-1.96) without any heterogeneity $\left(\mathrm{I}^{2}=0.0 \%, \mathrm{P}=0.900\right)$, which indicated that FGFR1 amplification was a negative predictor for DFS of ESCC patients (Figure 3).

\section{Correlations of FGFR1 amplification with clinicopathological parameters in resected ESCC}

The associations between FGFR1 amplification and main clinicopathologic features such as the gender, age, tumor depth, lymph node metastasis, metastasis, TNM stage, tumor differentiation status, history of drinking and smoking. Overall, the FGFR1 amplification was significantly associated with lymph node metastasis (N1-3 vs. N0) $(\mathrm{OR}=1.45,95 \% \mathrm{CI}: 1.13-1.86, \mathrm{P}=0.004)$, TNM stage (TNM III, IV vs. TNM I, II) $(\mathrm{OR}=1.33,95 \% \mathrm{CI}$ : 1.03-1.72, $\mathrm{P}=0.027$ ) and differentiation (moderate or poor vs. well) $(\mathrm{OR}=1.10,95 \% \mathrm{CI}: 1.07-1.13, \mathrm{P}<0.001)$. While, no significant correlation of FGFR1 amplification with sex (male $v s$. female), age ( $\geq 60 v s .<60)$, tumor depth (T3, $4 v s$. T1, 2), metastasis (positive vs. negative), drinking history (drinking $v s$. no drinking) or smoking history (smoking $v s$. no smoking) (Table 3).

\section{Sensitivity analysis}

To assess the stability of the pooled results, we conducted the sensitivity analysis; and it indicated that there was no single study which showed a significant influence on our 


Study
ID

Figure 2 Forest plot of the association between FGFR1 amplification and overall survival. FGFR1, fibroblast growth factor receptor 1; HR, hazard ratio; $\mathrm{CI}$, confidence interval.

Table 2 Meta-analyses for the association of FGFR1 amplification with survival of ESCC patients

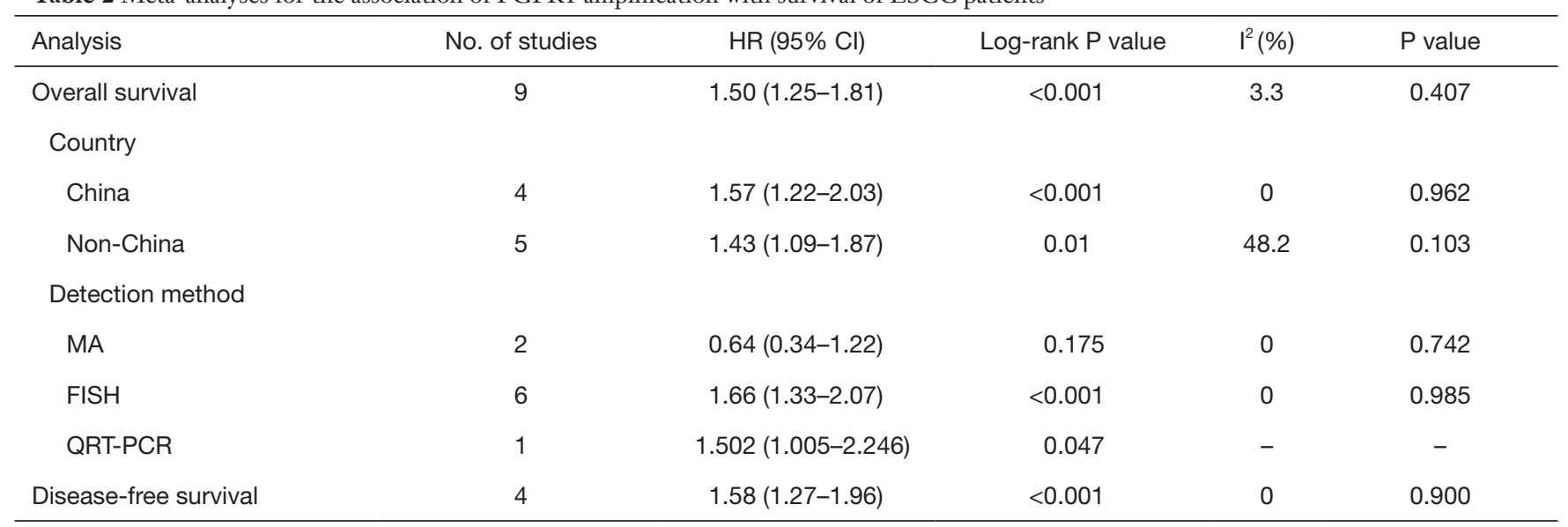

FGFR1, fibroblast growth factor receptor 1; ESCC, esophageal squamous cell carcinoma; HR, hazard ratio; Cl, confidence interval; MA, monoclonal antibody; FISH, fluorescent in situ hybridization; QRT-PCR, quantitative reverse transcription polymerase chain reaction.

results (Figure 4).

\section{Publication bias}

To detect potential publication bias, the Begg's funnel plot and Egger's test were both applied in our study. The Begg's funnel plot was relatively symmetrical $(\mathrm{P}=0.602)$ and the $\mathrm{P}$ value of Egger's test was 0.319 ; which manifested that no significant publication bias existed in our meta-analyses (Figure 5). 


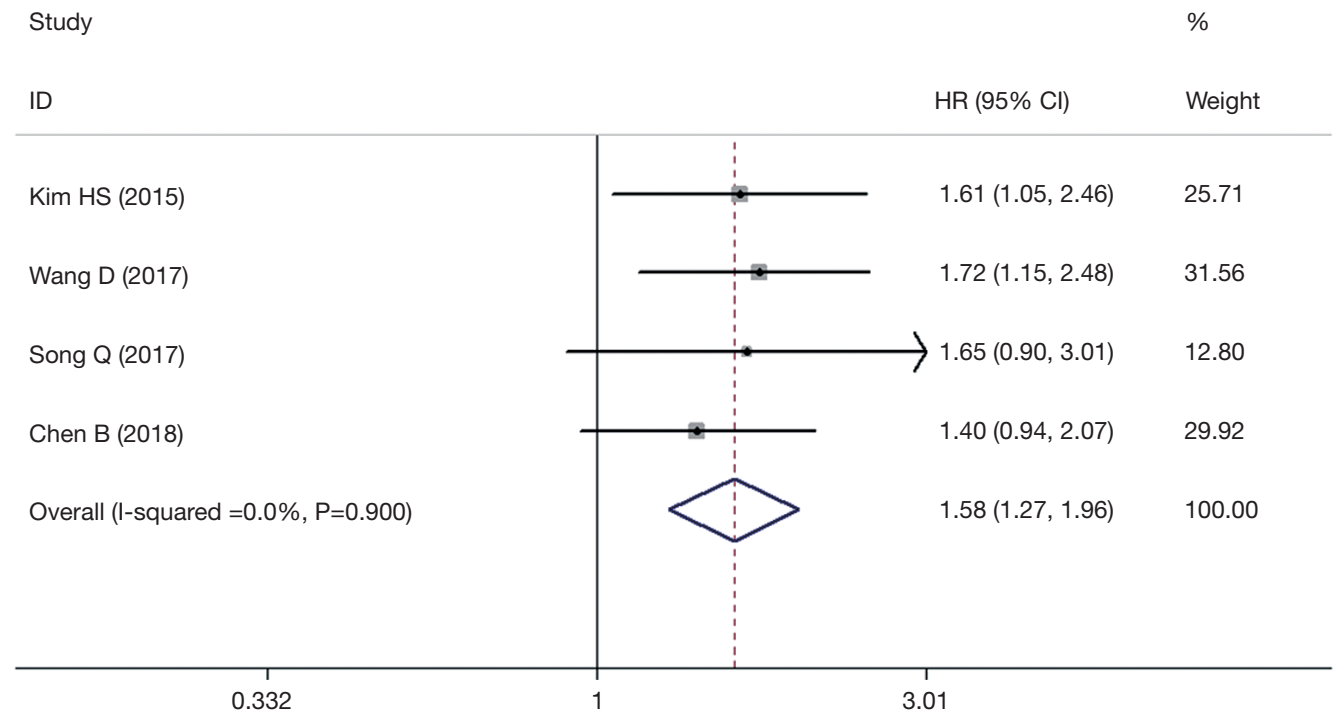

Figure 3 Forest plot of the association between FGFR1 amplification and disease-free survival. FGFR1, fibroblast growth factor receptor 1; HR, hazard ratio; CI, confidence interval.

Table 3 Correlations of FGFR1 amplification with clinicopathological characteristics

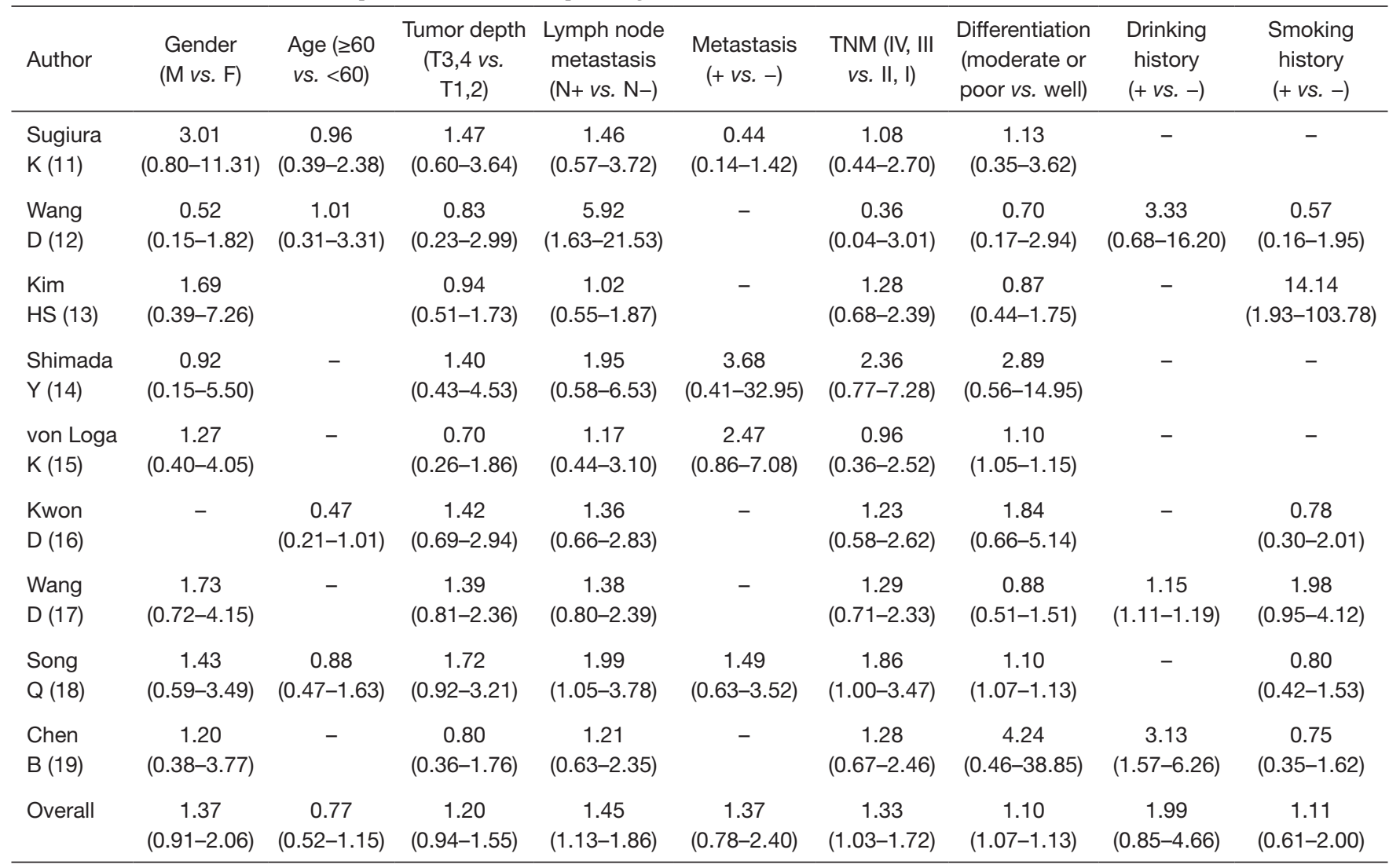

FGFR, fibroblast growth factor receptor; F, female; M, male; TNM, tumor-node-metastasis. 


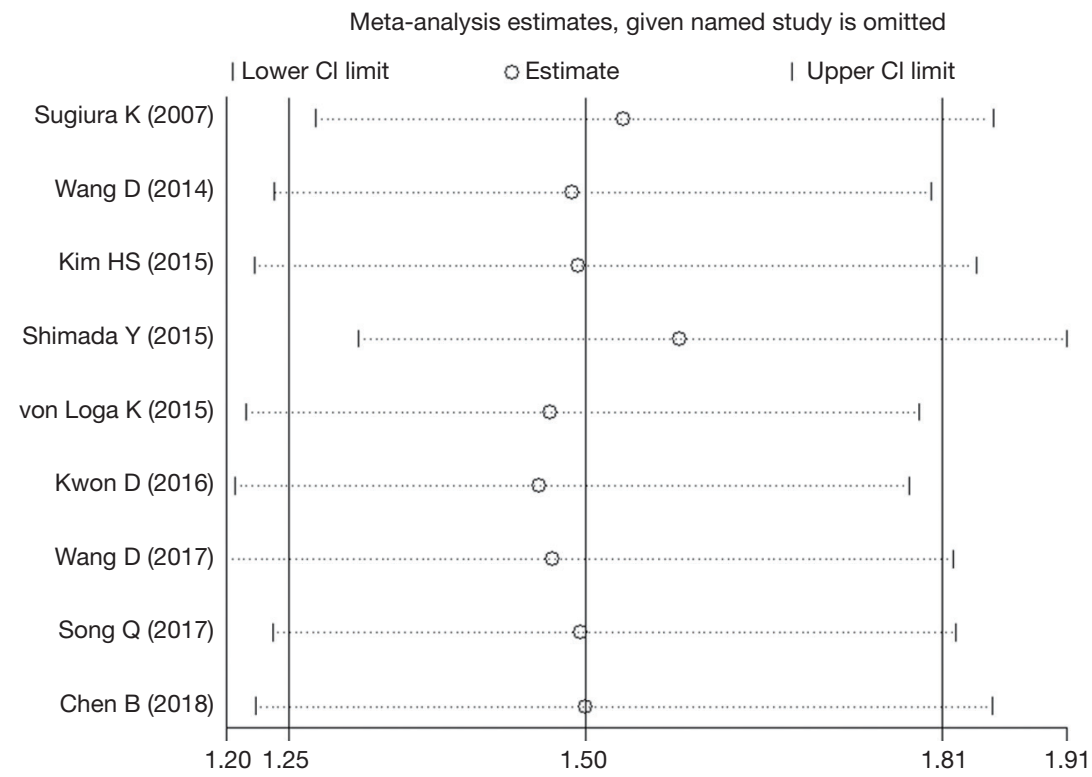

Figure 4 Sensitivity analysis of the association between FGFR1 amplification and overall survival. FGFR1, fibroblast growth factor receptor 1; CI, confidence interval.

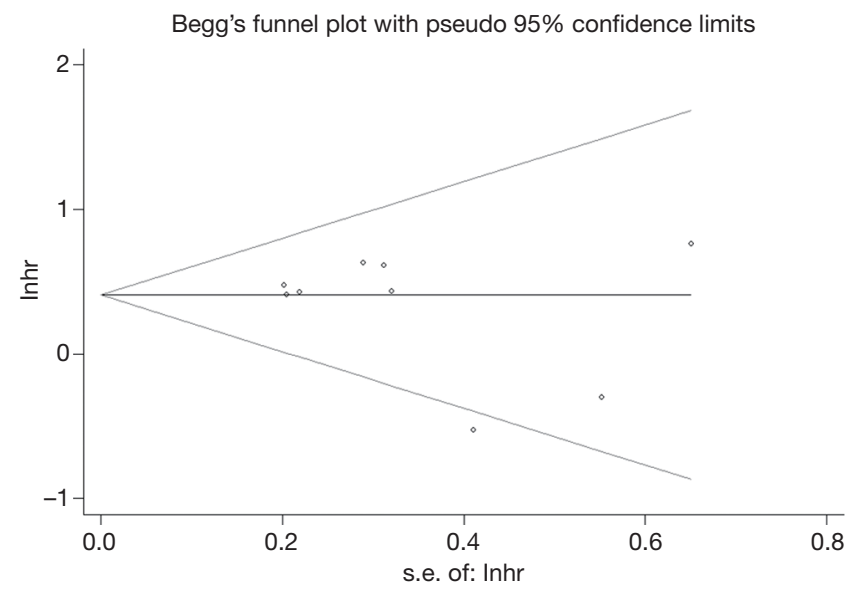

Figure 5 Begg's funnel plot of the association between FGFR1 amplification and overall survival. FGFR1, fibroblast growth factor receptor 1 .

\section{Discussion}

Esophageal cancer, as one of the most aggressive malignancies globally, consists of two main histological subtypes, ESCC and esophageal adenocarcinoma $(25,26)$. In Asian countries, ESCC accounts for the majority of all esophageal cancer cases $(25,26)$. Although there are several kinds of novel therapy methods such as the targeted therapy, the main treatments of ESCC are still traditional surgery and chemoradiotherapy and the prognosis remains poor.

Our research demonstrated that overexpression of FGFR1 gene was significantly associated with higher tumor stage, poorer differentiation and worse survival after the surgery in resected ESCC. According to previous studies, there are some potential mechanisms by which FGFR1 amplification is related to development and prognosis of ESCC. FGFR1, activated by fibroblast growth factor, was considered to play a role in provoking the signal transduction and activator of transcription (STAT) pathway directly or indirectly through Janus kinases (JAKs) and then inducing cellular proliferation and survival $(27,28)$. However, Chen et al. (19) reported that the inhibition of mitogenactivated protein kinase kinase (MEK)/extracellular signalregulated kinase (ERK) pathway may mostly contribute to suppression of FGFR1 expression and the MEK/ERK pathway may be the major signaling pathway which is mediated by FGFR1 in the progression of ESCC cells. Besides, another signaling pathway, epithelial-mesenchymal transition (EMT) which is modulated by the FGF1-FGFR1 axis, has been demonstrated to contribute to the metastasis of tumor cells in multiple cancers (29-31). Jiao et al. (29) found that after blocking the FGF1-FGFR1 axis through FGFR1 specific siRNAs, the role of FGF1 in promoting 
Cal27 cells migration and invasion abilities through the FGFR1 would be obviously inhibited, which indicated that FGFR1 amplification has an effect on the metastasis of tumor cells. Furthermore, FGFR1 was found to play a role in affecting the stem cell-like phenotype through regulating the expression and activity of GLI2 via the ERK pathway in lung SCC (32), which may indicate the role of FGFR1 gene in the differentiation of cancers; and our meta-analysis did demonstrate the significant association between FGFR1 gene amplification and differentiation in ESCC.

Based on these mechanisms mentioned above, some small molecular inhibitors targeting FGFR, such as the AZD4547, JNJ-42756493 and PRN1371, have been invented and applied in preclinical or clinical trials with obvious anti-tumor effect (33-37). However, there is no definite report that anti-FGFR1 therapy could beneficial ESCC patients with FGFR1 amplification significantly up to now.

Although there were a few articles which explored prognostic and clinicopathological significance of FGFR1 amplification in resected ESCC patients, their results were inconsistent. For example, among nine included studies, only three articles $(16,17,19)$ reported that FGFR1 amplification was an independent prognostic risk factor for ESCC patients and Song et al. (18) reported that FGFR1 amplification was significantly associated with TNM stage, which are consistent with our results. Therefore, the current meta-analysis is urgently needed to determine the association of FGFR1 with prognosis and clinicopathological parameters in resected ESCC and we did verify the significant correlation between FGFR1 amplification and lymph node metastasis status, TNM stage, differentiation status, OS and DFS of ESCC patients. We did not find the significant relation between FGFR1 amplification and drinking after combining the three eligible studies $(12,17,19)$, although Wang et al. (17) and Chen et al. (19) both indicated a significant correlation between drinking and FGFR1 amplification. Therefore, more researches are still needed to further testify the correlation of drinking and FGFR1 amplification. Similarly, Kim et al. (13) manifested a strongly significant association between smoking and FGFR1 amplification, but we came up with a negative result after combining several other studies which also reported negative results.

Actually, for FGFR1 amplification in ESCC, there are many fields which deserve further researches. First of all, how to predict the FGFR-targeted therapy response is still a big challenge for now, although increasing evidence indicates that FGFR1 mRNA expression may serve as a promising biomarker in predicting treatment outcomes of FGFR inhibitors (38). Whether the anti-FGFR therapy is suitable for patients who cannot receive the surgery due to the advanced tumor stage or other causes, better than chemoradiotherapies as postoperative adjuvant treatment or beneficial for patients recurrent ESCCs remains unclear. Besides, according to the results of our meta-analysis, FGFR1 amplification was not an independent prognostic factor for patients who were detected by the MA method $(16,19)$, but Sugiura et al. (11) and Shimada et al. (14) used different thresholds to define FGFR1 amplification. Therefore, it is necessary to compare the role of MA in predicting prognosis of resected ESCC patients with those of the other two methods and further determine definition of overexpression of FGFR1 gene when using the MA method.

There are some limitations in our study. First, all included studies are retrospective studies with relatively small sample sizes, which may cause some bias. Second, due to the lack of original data, we were unable to perform subgroup analyses for OS based on other important factors such as sex, age and TNM stage. Third, it is wellknown that the occurrence of ESCC is related to diet and race; unfortunately, none of included studies reported the association of FGFR1 amplification with these factors.

Inconclusion, out study demonstrated that FGFR1 amplification was significantly correlated with prognosis, tumor stage and differentiation status of resected ESCC patients. More well-designed prospective studies are still needed to clarify the real value of our findings.

\section{Acknowledgments}

None.

\section{Footnote}

Conflicts of Interest: The authors have no conflicts of interest to declare.

Ethical Statement: The authors are accountable for all aspects of the work in ensuring that questions related to the accuracy or integrity of any part of the work are appropriately investigated and resolved. All procedures performed in studies which involved human participants were in accordance with the ethical standards of the institutional and/or national research committee and with 
the 1964 Helsinki Declaration and its later amendments or comparable ethical standards.

\section{References}

1. Digklia A, Voutsadakis IA. Targeted treatments for metastatic esophageal squamous cell cancer. World J Gastrointest Oncol 2013;5:88-96.

2. Hubbard SR, Till JH. Protein tyrosine kinase structure and function. Annu Rev Biochem 2000; 69: 373-98.

3. Babina IS, Turner NC. Advances and challenges in targeting FGFR signalling in cancer. Nat Rev Cancer 2017;17:318-32.

4. Eswarakumar VP, Lax I, Schlessinger J. Cellular signaling by fbroblast growth factor receptors. Cytokine Growth Factor Rev 2005;16:139-49.

5. Grose R, Dickson C. Fibroblast growth factor signaling in tumorigenesis. Cytokine Growth Factor Rev 2005;16:179-86.

6. Helsten T, Elkin S, Arthur E, et al. The FGFR Landscape in Cancer: Analysis of 4,853 Tumors by Next-Generation Sequencing. Clin Cancer Res 2016;22:259-67.

7. Kim HR, Kim DJ, Kang DR, et al. Fibroblast growth factor receptor 1 gene amplification is associated with poor survival and cigarette smoking dosage in patients with resected squamous cell lung cancer. J Clin Oncol 2013;31:731-7.

8. von Mässenhausen A, Franzen A, Heasley L, et al. FGFR1 as a novel prognostic and predictive biomarker in squamous cell cancers of the lung and the head and neck area. Ann Transl Med 2013;1:23.

9. Brunello E, Brunelli M, Bogina G, et al. FGFR1 amplifcation in metastatic lymph-nodal and haematogenous lobular breast carcinoma. J Exp Clin Cancer Res 2012;31:103.

10. Xie FJ, Lu HY, Zheng QQ, et al. The clinical pathological characteristics and prognosis of FGFR1 gene amplification in non-small-cell lung cancer: a meta-analysis. Onco Targets Ther 2016;9:171-81.

11. Sugiura K, Ozawa S, Kitagawa Y, et al. Co-expression of aFGF and FGFR-1 is predictive of a poor prognosis in patients with esophageal squamous cell carcinoma. Oncol Rep 2007;17:557-64.

12. Wang D. The study of biological significance of fibroblast growth factor receptor 1 gene amplification in lung and esophageal squamous cell carcinoma D. Shandong: Shandong University, 2014:1-131.

13. Kim HS, Lee SE, Bae YS, et al. Fibroblast growth factor receptor 1 gene amplification is associated with poor survival in patients with resected esophageal squamous cell carcinoma. Oncotarget 2015;6:2562-72.

14. Shimada $Y$, Okumura T, Takei $Y$, et al. Role of fbroblast growth factor receptors in esophageal squamous cell carcinoma. Esophagus 2015;13:30-41.

15. von Loga K, Kohlhaussen J, Burkhardt L, et al. FGFR1 Amplification Is Often Homogeneous and Strongly Linked to the Squamous Cell Carcinoma Subtype in Esophageal Carcinoma. PLoS One 2015;10:e0141867.

16. Kwon D, Yun JY, Keam B, et al. Prognostic implications of FGFR1 and MYC status in esophageal squamous cell carcinoma. World J Gastroenterol 2016;22:9803-12.

17. Wang D, Du L, Wang Z, et al. Association of fibroblast growth factor receptor 1 gene amplification with poor survival in patients with esophageal squamous cell carcinoma. Oncotarget 2017;8:88857-69.

18. Song Q, Liu Y, Jiang D, et al. High amplification of FGFR1 gene is a delayed poor prognostic factor in early stage ESCC patients. Oncotarget 2017;8:74539-53.

19. Chen B, Liu S, Gan L, et al. FGFR1 signaling potentiates tumor growth and predicts poor prognosis in esophageal squamous cell carcinoma patients. Cancer Biol Ther 2018;19:76-86.

20. Stang A. Critical evaluation of the Newcastle-Ottawa scale for the assessment of the quality of nonrandomized studies in meta-analyses. Eur J Epidemiol 2010;25:603-5.

21. Tierney JF, Stewart LA, Ghersi D, et al. Practical methods for incorporating summary time-to-event data into metaanalysis. Trials 2007;8:16.

22. Lau J, Ioannidis JP, Schmid CH. Quantitative synthesis in systematic reviews. Ann Intern Med 1997;127:820-6.

23. Zintzaras E, Ioannidis JP. HEGESMA: genome search meta-analysis and heterogeneity testing. Bioinformatics 2005;21:3672-3.

24. Peters JL, Sutton AJ, Jones DR, et al. Comparison of two methods to detect publication bias in meta-analysis. JAMA 2006;295:676-80.

25. Ferlay J, Shin HR, Bray F, et al. Estimates of worldwide burden of cancer in 2008: GLOBOCAN 2008. Int J Cancer 2010;127:2893-917.

26. Torre LA, Bray F, Siegel RL, et al. Global cancer statistics, 2012. CA Cancer J Clin 2015;65:87-108.

27. Turner N, Grose R. Fibroblast growth factor signalling: from development to cancer. Nat Rev Cancer 2010;10:116-29.

28. Karajannis MA, Vincent L, Direnzo R, et al. Activation of FGFR1beta signaling pathway promotes survival, 
migration and resistance to chemotherapy in acute myeloid leukemia cells. Leukemia 2006;20:979-86.

29. Jiao J, Zhao X, Liang Y, et al. FGF1-FGFR1 axis promotes tongue squamous cell carcinoma (TSCC) metastasis through epithelial-mesenchymal transition (EMT). Biochem Biophys Res Commun 2015;466:327-32.

30. Tomlinson DC, Baxter EW, Loadman PM, et al. FGFR1induced epithelial to mesenchymal transition through MAPK/PLCgamma/COX-2-mediated mechanisms. PLoS One 2012;7:e38972.

31. Acevedo VD, Gangula RD, Freeman KW, et al. Inducible FGFR-1 activation leads to irreversible prostate adenocarcinoma and an epithelial-to-mesenchymal transition. Cancer Cell 2007;12:559-71.

32. Ji W, Yu Y, Li Z, et al. FGFR1 promotes the stem celllike phenotype of FGFR1-amplified non-small cell lung cancer cells through the Hedgehog pathway. Oncotarget 2016;7:15118-34.

33. Nogova L, Sequist LV, Perez Garcia JM, et al. Evaluation of BGJ398, a fibroblast growth factor receptor 1-3 Kinase inhibitor, in patients with advanced solid tumors harboring genetic alterations in fibroblast growth factor receptors: results of a global Phase I, dose-escalation and dose-

Cite this article as: Wang Y, Wu Y, Li J, Lai Y, Zhou K, Che G. Prognostic and clinicopathological significance of FGFR1 gene amplification in resected esophageal squamous cell carcinoma: a meta-analysis. Ann Transl Med 2019;7(22):669. doi: 10.21037/ atm.2019.10.69 expansion study. J Clin Oncol 2017;35:157-65.

34. Tabernero J, Bahleda R, Dienstmann R, et al. Phase I DoseCANCER BIOLOGY \& THERAPY 85 escalation study of JNJ-42756493, an oral pan-fibroblast growth factor receptor inhibitor, in patients with advanced solid tumors. J Clin Oncol 2015;33:3401-8.

35. Papadopoulos KP, Tolcher AW, Patnaik A, et al. Phase 1, first-in-human study of ARQ 087, an oral panFibroblast Growth Factor Receptor (FGFR) inhibitor, in patients (pts) with advanced solid tumors. J Clin Oncol 2017;35:abstr 4017.

36. Smyth EC, Turner NC, Peckitt C, et al. Phase II multicenter proof of concept study of AZD4547 in FGFR amplified tumours. J Clin Oncol 2015;33:abstr 2508.

37. Andre F, Ranson M, Dean E, et al. Abstract LB-145: Results of a phase I study of AZD4547, an inhibitor of fibroblast growth factor receptor (FGFR), in patients with advanced solid tumors. Cancer Res 2013;73:abstr LB-145.

38. Göke F, Franzen A, Hinz TK, et al. FGFR1 expression levels predict BGJ398 sensitivity of FGFR1-dependent head and neck squamous cell cancers. Clin Cancer Res 2015;21:4356-64. 